\title{
The PRIMARA study: a prospective, descriptive, observational study to review cinacalcet use in patients with primary hyperparathyroidism in clinical practice
}

\author{
P Schwarz, J J Body ${ }^{1}$, J Cáp ${ }^{2}$, L C Hofbauer ${ }^{3}$, M Farouk ${ }^{4}$, A GessI ${ }^{5}$, J M Kuhn ${ }^{6}$, C Marcocci ${ }^{7}$, \\ C Mattin ${ }^{8}$, M Muñoz Torres $^{9}$, J Payer ${ }^{10}$, A Van De Ven ${ }^{11}$, M Yavropoulou ${ }^{12}$ and P Selby ${ }^{13}$ \\ Department of Medicine, Copenhagen University Hospital Glostrup, Ndr. Ringvej 69, Building 88, 2600 Glostrup, \\ Denmark, 'Department of Medicine, CHU Brugmann, Université Libre de Bruxelles, Place Van Gehuchten 4, \\ 1020 Brussels, Belgium, ${ }^{2} 4$ th Department of Internal Medicine, University Hospital of Hradec Králové, 50005 Hradec \\ Králové, Czech Republic, ${ }^{3}$ Endokrinologie, Diabetes und Osteologie, Technische Universität Dresden, Fetscherstrasse \\ 74, D-01307 Dresden, Germany, ${ }^{4}$ Nephrology Therapeutic Area, Amgen Europe GmbH, Dammstrasse 23, \\ CH-6301 Zug, Switzerland, ${ }^{5}$ Division of Endocrinology and Metabolism, Medical University of Vienna, Währinger \\ Gürtel 18-20, 1090 Vienna, Austria, ${ }^{6}$ Service d'Endocrinologie, Diabète et Maladies Métaboliques, University \\ Hospital of Rouen, Hôpital de Bois Guillaume, 147 Avenue du Maréchal Juin, 76230 Bois-Guillaume Cedex, France, \\ ${ }^{7}$ Department of Clinical and Experimental Medicine, University of Pisa, Via Paradisa 2, 56124 Pisa, Italy, \\ ${ }^{8}$ Global Biostatistical Science, Amgen Ltd, 240 Cambridge Science Park, Milton Road, Cambridge CB4 OWD, UK, \\ ${ }^{9}$ Endocrinology Division, University Hospital San Cecilio, Avenida Dr Olóriz 16, 18012 Granada, Spain, \\ ${ }^{10} 5$ th Department of Internal Medicine, Faculty Hospital Bratislava, University Hospital of Bratislava, Ruzinovska 6, \\ 82606 Bratislava, Slovakia, ${ }^{11}$ Department of General Internal Medicine, Radboud University Nijmegen Medical \\ Centre, PO Box 9101, 6500 HB Nijmegen, The Netherlands, ${ }^{12}$ Department of Endocrinology and Metabolism, AHEPA \\ University Hospital, Aristotle University of Thessaloniki, 1 Stilponos Kyriakidi Street, 54636 Thessaloniki, Greece and \\ ${ }^{13}$ Department of Medicine, Institute of Human Development, Manchester Royal Infirmary, University of \\ Manchester, Oxford Road, Manchester M13 9WL, UK
}

Correspondence should be addressed to P Schwarz Email peter.schwarz@regionh.dk

\section{Abstract}

Objective: Medical management of primary hyperparathyroidism (PHPT) is important in patients for whom surgery is inappropriate. We aimed to describe clinical profiles of adults with PHPT receiving cinacalcet. Design: A descriptive, prospective, observational study in hospital and specialist care centres.

Methods: For patients with PHPT, aged 23-92 years, starting cinacalcet treatment for the first time, information was collected on dosing pattern, biochemistry and adverse drug reactions (ADRs). Initial cinacalcet dosage and subsequent dose changes were at the investigator's discretion.

Results: Of 303 evaluable patients with PHPT, 134 (44\%) had symptoms at diagnosis (mostly bone pain (58) or renal stones (50)). Mean albumin-corrected serum calcium (ACSC) at baseline was $11.4 \mathrm{mg} / \mathrm{dl}(2.9 \mathrm{mmol} / \mathrm{l})$. The reasons for prescribing cinacalcet included: surgery deemed inappropriate (35\%), patient declined surgery (28\%) and surgery failed or contraindicated $(22 \%)$. Mean cinacalcet dose was $43.9 \mathrm{mg} /$ day (s.D., 15.8) at treatment start and $51.3 \mathrm{mg} / \mathrm{day}(31.8)$ at month 12; 219 (72\%) patients completed 12 months treatment. The main reason for cinacalcet discontinuation was parathyroidectomy (40; 13\%). At 3, 6 and 12 months from the start of treatment, 63,69 and $71 \%$ of patients, respectively, had an ACSC of $\leq 10.3 \mathrm{mg} / \mathrm{dl}$ vs $9.9 \%$ at baseline. Reductions from baseline in ACSC of $\geq 1 \mathrm{mg} / \mathrm{dl}$ were seen in 56,63 and $60 \%$ of patients respectively. ADRs were reported in 81 patients ( $27 \%$ ), most commonly nausea. A total of $7.6 \%$ of patients discontinued cinacalcet due to ADRs. Conclusions: Reductions in calcium levels of $\geq 1 \mathrm{mg} / \mathrm{dl}$ was observed in $60 \%$ of patients 12 months after initiation of cinacalcet, without notable safety concerns. 


\section{Introduction}

Primary hyperparathyroidism (PHPT) is characterised by elevated secretion of parathyroid hormone (PTH) leading to hypercalcaemia, which can reach levels that are detrimental to health (1). Although the disease is often asymptomatic, complications can be progressive and/or severe, including bone loss and fractures, kidney stones and gastrointestinal disturbances; symptoms such as muscle weakness, depression, and neuropsychiatric disturbance have also been reported. The only definitive treatment for PHPT is parathyroidectomy, but surgery may be contraindicated in some patients, for example, because of advanced age or the presence of co-morbidities (2).

Cinacalcet is a calcimimetic, a class of drugs that reduce PTH secretion by amplifying the sensitivity of the calcium-sensing receptor. These receptors are found mainly in the parathyroid glands and the kidney, where they regulate extracellular calcium $(3,4,5)$. Cinacalcet is the first of this drug class to be introduced and is available in over 55 countries. In most countries, the three licensed indications are the treatment of secondary HPT in patients with chronic kidney disease receiving dialysis, the treatment of hypercalcaemia in patients with parathyroid carcinoma, and the treatment of hypercalcaemia in PHPT in patients for whom parathyroidectomy is not a treatment option.

PRIMARA (a prospective, descriptive, observational study to review Mimpara (cinacalcet) use in primary hyperparathyroidism in clinical practice) was an observational study whose overall objective was to describe the demographic and clinical profiles of adults who were prescribed cinacalcet for PHPT in clinical practice, and the treatment patterns employed. The study, which enrolled patients at several European sites, also aimed to examine outcomes in treated patients, including levels of serum calcium and other laboratory parameters as well as the incidence of adverse drug reactions (ADRs).

\section{Subjects and methods}

\section{Study conduct}

PRIMARA (NCT 00928408) was a prospective multicentre observational study. The patients were enrolled from October 2009 to December 2010 at 60 hospitals and specialist care centres across 12 European countries. The observational period was 1 year from enrollment. This study was conducted in compliance with the principles of the Declaration of Helsinki and its amendments, and in accordance with International Conference on Harmonisation Good Clinical Practice guidelines. The study protocol was approved by independent Ethics Committees at each study site, and patients provided informed consent where required by local law.

\section{Patient selection and treatment}

To be eligible for inclusion, patients with PHPT had to be $\geq 18$ years of age at the time of starting cinacalcet treatment and had to be enrolled no later than 1 month after starting cinacalcet treatment. The patients were ineligible if they had received previous cinacalcet treatment (other than in the period immediately before study entry), had a diagnosis of secondary HPT or had hypercalcaemia of other aetiology, e.g. cancer or sarcoidosis. No criteria were predefined for diagnosing PHPT; this was left to the investigator's judgement. Prescription of cinacalcet that did not comply with European Medicines Agency labelling was not a reason for exclusion per se. The dosage of cinacalcet during the study period was not predefined: study centres could alter dosage at any time during this period.

Study centres with experience in the treatment of PHPT and cinacalcet use were selected. The reasons for non-enrollment were recorded to assess possible selection bias for all patients who received cinacalcet at any of the study centres but were not enrolled in the study.

\section{Data collection and assessments}

Baseline data (see Statistical analyses section for definition), and data measured after cinacalcet treatment had started but before patient enrollment, were abstracted from medical records to electronic case report forms (eCRFs) within 4 weeks of enrollment. All other study data were abstracted in the same way (within 4 weeks of the assessment) for up to 12 months after patient enrollment. Every effort was made to collect the followup data for patients who discontinued cinacalcet before the end of the 12-month observational period.

Cinacalcet is administered orally; data were collected on the dose of cinacalcet per administration (mg), the frequency of cinacalcet dosing and the reason for any dose changes. The investigator's reason for prescribing cinacalcet was also recorded. Data on concentrations of serum calcium (total, albumin-corrected and ionised, 
as available), plasma intact PTH (iPTH) and phosphorus and on the occurrence of parathyroidectomy were also collected. The methods used to derive these measurements at each site are not described because of the large number of participating sites and the variety of methods and assays being used.

The ADRs, i.e. adverse events considered by the investigator to be causally associated with cinacalcet administration, were collected.

\section{Statistical analyses}

The full analysis set (FAS) included all enrolled patients who fulfilled the study inclusion/exclusion criteria (see Patient selection and treatment section): unless otherwise stated, results reported are for the FAS. Some analyses were repeated on a second analysis set, 'FAS with Censoring', which excluded data obtained after discontinuation of cinacalcet. Analysis was based on available data with no imputation of missing values.

Descriptive analysis was performed. For continuous variables, the following statistics are reported as applicable: mean, s.D., S.E.M., 95\% CI for the mean, median, first and third quartiles (Q1 and Q3), and minimum and maximum values. For categorical variables, the number and proportion (\%) of patients in each category are reported together with 95\% CIs. Because of the descriptive nature of the study, no hypothesis testing was performed and differences compared with baseline were not tested for statistical significance.

Cinacalcet dose and frequency data were summarised at treatment initiation and after 3, 6 and 12 months of treatment (and on the day that treatment stopped, if earlier than 12 months). If a dose was withheld at a given time point, it was included as a dose of zero. The changes in dosage throughout the study (amount and/or frequency) were summarised in three time periods: $0-3$ months, $>3$ and $\leq 6$ months, and more than 6 months from treatment start. The mean daily dose and duration of exposure to cinacalcet over the entire study were determined; the duration of exposure was defined as the time from first dose to the last non-zero dose.

For biochemical parameters, the most recent value in the 3 months before the start of cinacalcet treatment was used as the baseline value, and change from baseline was calculated at 3, 6 and 12 months after starting treatment. In addition, we derived the proportion of patients with albumin-corrected serum calcium (ACSC) concentrations of $\leq 10.3 \mathrm{mg} / \mathrm{dl}(2.6 \mathrm{mmol} / \mathrm{l})$ at 3,6 and 12 months and the proportion who achieved a reduction from baseline of ACSC of $\geq 1 \mathrm{mg} / \mathrm{dl}(0.25 \mathrm{mmol} / \mathrm{l})$ at the same time points. Where possible, ACSC values were calculated from total calcium and albumin values (if collected within 7 days of one another); if not, albumin-corrected values provided by the site were used in the analysis. The following formula was used to calculate corrected calcium values if albumin was $<40 \mathrm{~g} / \mathrm{l}(4 \mathrm{~g} / \mathrm{dl})$ (6): corrected calcium $(\mathrm{mmol} / \mathrm{l})=$ total calcium $(\mathrm{mmol} / \mathrm{l})+$ $0.02(40$ - serum albumin $(\mathrm{g} / \mathrm{l}))$. If albumin was $\geq 40 \mathrm{~g} / \mathrm{l}$, the corrected calcium value $(\mathrm{mmol} / \mathrm{l})$ was considered to be equivalent to total calcium ( $\mathrm{mmol} / \mathrm{l})$.

As this was an observational study, there was no guarantee that patients would have assessments at the predefined analysis time points, e.g. 3 months after starting cinacalcet treatment. For biochemical data, therefore, the closest assessment to each analysis time point was used for analysis, within the following time windows: month $3=$ from 0 to 4.5 months after treatment start; month $6=$ from 4.5 to 9 months after treatment start; month $12=$ from 9 to 13 months after treatment start.

\section{Results}

\section{Patient disposition}

A total of 305 patients were enrolled in the study, 303 of whom were included in the FAS: two patients were excluded because they started cinacalcet treatment outside the permitted 1-month enrollment window. A total of 282 patients completed the 12-month observation period, 219 of whom were still receiving cinacalcet at the end of the 12-month observation period.

Eighty-six patients $(28.2 \%)$ discontinued cinacalcet during the study, most commonly because of parathyroidectomy $(40 ; 13.1 \%)$; the median treatment duration in these 40 patients was 86.5 days (range, 4-330 days). Cinacalcet was discontinued because of nausea and/or vomiting in six patients $(2.0 \%)$. Other reasons for discontinuation of cinacalcet included death $(n=4)$, hypocalcaemia $(n=3)$, lost to follow-up $(n=3)$, noncompliance $(n=2)$, poor response $(n=2)$, high PTH $(n=1)$, consent withdrawn $(n=1)$ and other $(n=24)$.

\section{Baseline characteristics}

The main demographic and clinical characteristics of the patients are given in Table 1 . The majority of patients were female $(241 / 303,79.5 \%)$. The median age of patients at the time of starting cinacalcet treatment was 70 (range, $23-92$ ) years, and $64 \%$ of patients were $\geq 65$ years of age. 
Table 1 Baseline demographics and clinical characteristics in full analysis set (FAS).

\begin{tabular}{|c|c|}
\hline & $\begin{array}{l}\text { All patients in FAS } \\
\qquad(n=303)\end{array}$ \\
\hline Female patients, $n(\%)$ & $241(79.5)$ \\
\hline Median age, years (range) & $70(23-92)$ \\
\hline Aged $\geq 65$ years, $n(\%)$ & $194(64.0)$ \\
\hline $\begin{array}{l}\text { Median time since diagnosis, } \\
\text { years (Q1 and Q3) }\end{array}$ & $0.55(0.10,2.42)$ \\
\hline \multicolumn{2}{|l|}{ Primary aetiology of $\mathrm{PHPT}^{\mathrm{a}}, n(\%)$} \\
\hline Parathyroid adenoma & $224(73.9)$ \\
\hline Parathyroid hyperplasia & $49(16.2)$ \\
\hline Parathyroid carcinomas & $2(0.7)$ \\
\hline Unknown & $28(9.2)$ \\
\hline Parathyroidectomised, $n(\%)$ & $45(14.9)$ \\
\hline History of renal disease, $n(\%)$ & $49(16.2)$ \\
\hline History of kidney stones, $n(\%)$ & $66(21.8)$ \\
\hline History of cardiovascular disease ${ }^{\mathrm{b}}, n(\%)$ & $78(25.7)$ \\
\hline \multicolumn{2}{|l|}{$\begin{array}{l}\text { Biochemistry values (mean and S.D. } \\
\text { unless stated) }\end{array}$} \\
\hline Serum total calcium, $\mathrm{mmol} / \mathrm{l}(n=275)$ & $2.84(0.32)$ \\
\hline $\begin{array}{l}\text { Albumin-corrected serum calcium, } \\
\mathrm{mmol} / \mathrm{l}(n=198)\end{array}$ & $2.86(0.26)$ \\
\hline $\begin{array}{l}\text { Serum/plasma iPTH, pmol/l }(n=258) \\
\text { (median, Q1 and Q3) }\end{array}$ & $16.20(11.14,27.91)$ \\
\hline Serum phosphorus, $\mathrm{mmol} / \mathrm{l}(n=245)$ & $0.84(0.26)$ \\
\hline Serum $25-\mathrm{OH}$ vitamin $\mathrm{D}, \mathrm{nmol} / \mathrm{l}(n=189)$ & $47.5(26.3)$ \\
\hline Serum creatinine, $\mu \mathrm{mol} / \mathrm{l}(n=253)$ & $82.6(35.2)$ \\
\hline Alkaline phosphatase, IU/I $(n=213)$ & $110.6(71.6)$ \\
\hline
\end{tabular}

iPTH, intact parathyroid hormone; PHPT, primary hyperparathyroidism; Q1 and Q3, first and third quartiles.

a Taken from electronic case report form responses (physicians asked to select one of the listed options).

${ }^{b}$ Other than hypertension.

The median (Q1 and Q3) time since PHPT diagnosis was $0.55(0.10,2.42)$ years, diagnosis being symptom-based, as opposed to based solely on PTH and calcium values, in 134 (44.2\%) patients; the most common diagnostic symptoms in this group of patients were bone pain $(58 / 134,43.3 \%)$ and kidney stones $(50 / 134,37.3 \%)$. In most cases, the primary aetiology of PHPT was reported as parathyroid adenomas $(73.9 \%$ of patients), although this was not histologically proven. Forty-five patients (14.9\%) had previously undergone parathyroidectomy.

The majority of patients were prescribed cinacalcet because surgery was not considered appropriate by their physician $(107 / 303,35.3 \%)$ or the patient declined surgery (86/303, 28.4\%). Other reasons included acute or chronic use of cinacalcet for treatment of hypercalcaemia before surgery (off-label indication) (43/303, 14.2\%), previous surgery failure $(33 / 303,10.9 \%)$ and surgery being contraindicated (33/303, 10.9\%). In the 107 patients for whom surgery was not considered appropriate by their physician, the most common single reason was that the diseased parathyroid gland could not be located by imaging techniques $(45 / 107,42.1 \%)$; other reasons included age and co-morbidities.

Patients who received cinacalcet as an alternative to surgery (because of contraindications, inappropriateness or lack of consent) were slightly older than patients who received it after surgery failure or as presurgical treatment (median ages: 76.0, 71.0 and 70.5 years, and 62.0 and 64.0 years respectively).

\section{Cinacalcet dosage and treatment pattern}

The number of patients receiving cinacalcet at each time point was $303(100 \%)$ at initiation, 255 (84\%) at 3 months, $233(77 \%)$ at 6 months and $219(72 \%)$ at 12 months. Most patients started at a dose of either $30 \mathrm{mg}$ once daily (53.8\%) or $30 \mathrm{mg}$ twice daily (43.6\%), and these continued to be the most common dosing frequencies during the study; the mean and median daily cinacalcet dose at each time point is given in Table 2. At the time of the last recorded treatment (including those who discontinued treatment), $55.4 \%(168 / 303)$ of patients were taking cinacalcet once daily and $38.9 \%(118 / 303)$ were taking it twice daily, and the mean daily dose was $53.1 \mathrm{mg}$ (s.E.M., $2.0 \mathrm{mg}$ ). The median dose was $30 \mathrm{mg} /$ day.

For 148 patients $(48.8 \%)$, the dose and/or dosing frequency changed at least once during the study. For those with a dosing change, reasons included hypercalcaemia $(52.7 \%)$, cinacalcet restarted $(22.3 \%)$, nausea and/or vomiting (18.9\%), hypocalcaemia (12.2\%) and high PTH levels (10.1\%).

The mean (s.E.m.) daily cinacalcet dose during the whole study period was $49.6 \mathrm{mg}(1.6 \mathrm{mg})$, including periods when drug was withheld, and $50.4 \mathrm{mg}(1.6 \mathrm{mg})$ otherwise. Based on the data collected during the study, the mean (s.E.M.) duration of exposure was 295.9 days (7.3 days) and the median was 366 days (range, 2-397). Nineteen patients $(6.3 \%)$ received treatment for $<1$ month, and 31 (10.2\%) for 1-3 months. Two hundred and twenty-two patients (73.3\%) received cinacalcet for more than 9 months.

\section{Calcium outcomes}

At baseline, 22/223 (9.9\%) patients had an ACSC concentration of $\leq 10.3 \mathrm{mg} / \mathrm{dl} \quad(\leq 2.6 \mathrm{mmol} / \mathrm{l})$. The proportion of patients achieving this target at 3, 6 and 12 months was $62.7,69.4$ and $70.9 \%$ respectively (Fig. 1a). The results were very similar in the FAS-with-Censoring analysis set, which excluded data after cinacalcet discontinuation: the proportions were 61.0, 69.5 and $71.4 \%$ 
Table 2 Dosing summary for cinacalcet at each time point (full analysis set).

\begin{tabular}{|c|c|c|c|c|}
\hline \multirow[b]{2}{*}{ Parameter } & \multicolumn{4}{|c|}{ Number of patients (\%) } \\
\hline & $\begin{array}{l}\text { Initiation } \\
(n=303)\end{array}$ & $\begin{array}{l}\text { Month } 3 \\
(n=255)\end{array}$ & $\begin{array}{l}\text { Month } 6 \\
(n=233)\end{array}$ & $\begin{array}{l}\text { Month 12 } \\
(n=219)\end{array}$ \\
\hline \multicolumn{5}{|l|}{ Daily dose (mg) } \\
\hline Mean (s.E.M.) & $43.9(0.9)$ & $48.7(1.9)$ & $48.9(2.0)$ & $51.3(2.1)$ \\
\hline Median (range) & $30(15-120)$ & $30(0-240)$ & $30(0-240)$ & $30(9-240)^{a}$ \\
\hline \multicolumn{5}{|l|}{ Dosage taken $(n, \%)$} \\
\hline $30 \mathrm{mg}$ once daily & $163(53.8)$ & $126(49.4)$ & $120(51.5)$ & $108(49.3)$ \\
\hline $30 \mathrm{mg}$ twice daily & $132(43.6)$ & 89 (34.9) & $74(31.8)$ & $64(29.2)$ \\
\hline $60 \mathrm{mg}$ once daily & $4(1.3)$ & $3(1.2)$ & $2(0.9)$ & $4(1.8)$ \\
\hline 60 mg twice daily & $1(0.3)$ & $12(4.7)$ & $14(6.0)$ & $16(7.3)$ \\
\hline Other dosages & $3(1.0)$ & $19(7.5)$ & $20(8.6)$ & $27(12.3)$ \\
\hline Dose withheld & $0(0)$ & $6(2.4)$ & $3(1.3)$ & $0(0)$ \\
\hline
\end{tabular}

respectively. In an additional analysis that excluded the 22 patients with baseline ACSC concentrations of $\leq 10.3 \mathrm{mg} / \mathrm{dl}$, the proportion of patients achieving the target concentration was $60.1,67.8$ and $70.1 \%$ at 3,6 and 12 months respectively.

After 3 months, 101/180 (56.1\%) patients achieved a reduction from baseline in ACSC of $\geq 1 \mathrm{mg} / \mathrm{dl}$ ( $\geq 0.25 \mathrm{mmol} / \mathrm{l})$. At 6 and 12 months, the corresponding proportions were 95/152 (62.5\%) and 70/117 (59.8\%) respectively (Fig. 1b). The results were similar in the FASwith-Censoring analysis set: percentages were 57.3, 64.5 and $62.0 \%$ respectively. After excluding the 22 patients whose baseline ACSC concentration was $\leq 10.3 \mathrm{mg} / \mathrm{dl}$, the proportion of patients achieving the reduction target was $60.7,67.2$ and $64.5 \%$ at 3,6 and 12 months respectively.

ACSC concentrations at each time point are given in Table 3 and Fig. 2a; mean (s.E.M.) values fell from the baseline value of $11.42(0.07) \mathrm{mg} / \mathrm{dl}(2.86(0.02) \mathrm{mmol} / \mathrm{l})$ to $10.12(0.08) \mathrm{mg} / \mathrm{dl}(2.53(0.02) \mathrm{mmol} / \mathrm{l})$ at month 3 (mean decrease by $11.0 \%$ ) and showed little change thereafter. Data for total calcium were available for a larger number of patients than data for ACSC, but the pattern of results for the two measures was similar: the mean and S.E.M. values at baseline $(n=275)$ and 3 months $(n=268)$ were $11.34(0.08) \mathrm{mg} / \mathrm{dl}(2.84(0.02) \mathrm{mmol} / \mathrm{l})$ and $9.92(0.07) \mathrm{mg} / \mathrm{dl}(2.48(0.02) \mathrm{mmol} / \mathrm{l})$ respectively. The results were similar in the FAS-with-Censoring analysis set.

\section{Other laboratory values}

The changes in concentrations of iPTH and phosphorus from baseline at each study time point are given in Fig. 2b and c and Supplementary Table 1, see section on supplementary data given at the end of this article.
After cinacalcet initiation, reductions in iPTH and increases in phosphorus concentrations were observed (mean changes from baseline of -8 to $-13 \%$ and approximately $+20 \%$ respectively).

\section{Surgery during study}

Forty-five patients underwent parathyroidectomy during the study, of whom 43 discontinued cinacalcet beforehand. In 27 (60\%) of those who had surgery, cinacalcet was originally prescribed for the reduction in hypercalcaemia before surgery, and in 14 others, it was prescribed because surgery was declined by the patient or considered inappropriate by the physician. The reasons in the other four patients were failed surgery $(n=2)$, surgery contraindicated $(n=1)$ and other $(n=1)$. No data were collected on surgical outcomes.

\section{Tolerability and safety}

ADRs were reported in 81/303 (26.7\%) patients. The most common ADRs are listed in Table 3 by preferred term. Nausea was the most frequently reported ADR reported in $41 / 303(13.5 \%)$ patients. Vomiting was reported in 11 (3.6\%) patients, and abdominal pain and diarrhoea were each reported in $6(2.0 \%)$ patients. Hypocalcaemia and paraesthaesia were each reported in five (1.7\%) patients, with one patient reporting both hypocalcaemia and paraesthaesia: of the nine patients affected by these two ADRs, baseline corrected calcium concentrations were $<10.3 \mathrm{mg} / \mathrm{dl}(<2.6 \mathrm{mmol} / \mathrm{l})$ in two, and a third had a total baseline calcium value of $8.91 \mathrm{mg} / \mathrm{dl}$ (but no corrected calcium value). 

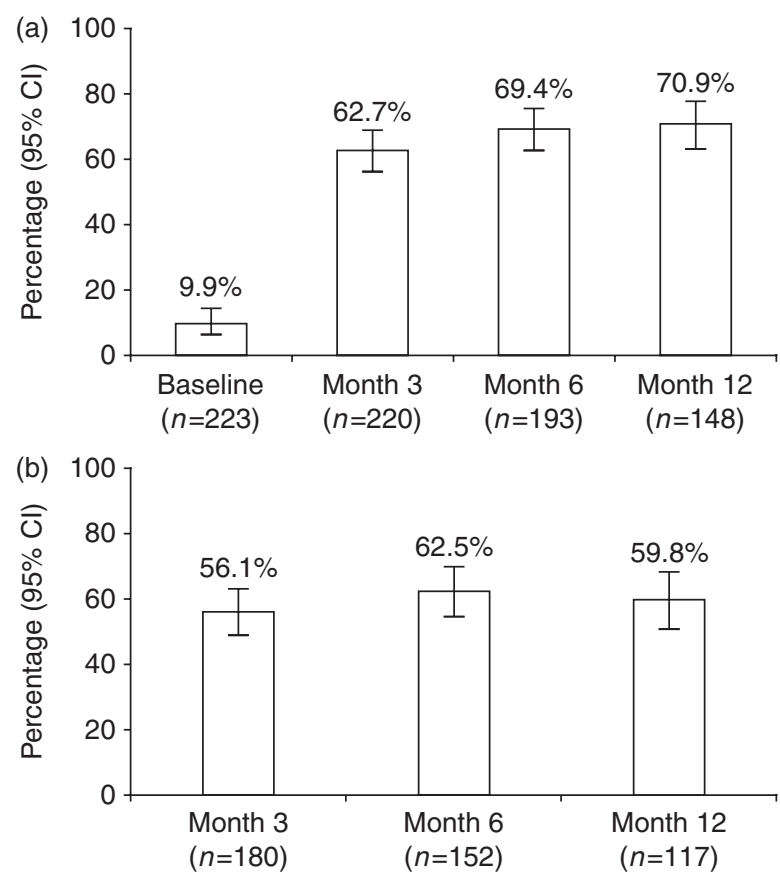

\section{Figure 1}

Reductions in calcium levels during the study in the full analysis set: (a) proportion of patients achieving an albumin-corrected serum calcium concentration of $\leq 10.3 \mathrm{mg} / \mathrm{dl}$ ( $\leq 2.6 \mathrm{mmol} / \mathrm{l})$ and (b) percentage of patients achieving a reduction in albumincorrected serum calcium concentrations of $\geq 1 \mathrm{mg} / \mathrm{dl}$ ( $\geq 0.25 \mathrm{mmol} / \mathrm{l}$ ). If an albumin-corrected calcium value could not be calculated or was not provided by the site, an ionised calcium value was used instead (using ionised calcium thresholds of $5.3 \mathrm{mg} / \mathrm{dl}(1.32 \mathrm{mmol} / \mathrm{l})$ for the first outcome and $1 \mathrm{mg} / \mathrm{dl}(0.25 \mathrm{mmol} / \mathrm{l})$ for the second). Error bars $=95 \% \mathrm{Cls}$.

Three serious ADRs were reported in two patients: hypocalcaemia and muscle spasms (one patient receiving cinacalcet $60 \mathrm{mg}$ once daily) and circulatory collapse (one patient receiving cinacalcet $30 \mathrm{mg}$ twice daily). These occurred 2-3 weeks after the start of cinacalcet treatment; in both cases, the ADRs resolved after drug was discontinued. ADRs leading to discontinuation were reported in 23 (7.6\%) patients: the most frequent were nausea (seven patients, $2.3 \%$ ), vomiting (five patients, 1.7\%) and paraesthaesia (three patients, 1.0\%). Decreased appetite, hypocalcaemia, headache and tremor each occurred in two patients, and other ADRs leading to discontinuation were each reported in one patient only. No patients experienced life-threatening or fatal ADRs; when summarised by highest severity grade, most patients experienced ADRs that were mild or moderate in severity.

\section{Discussion}

PRIMARA is the first study of its type to investigate cinacalcet use in patients with PHPT in clinical practice across Europe, and provides valuable insights into the type of patients for whom cinacalcet treatment is considered appropriate and what dosing regimens are typically employed. The majority of the PHPT patients in our sample were being treated with cinacalcet as an alternative for undergoing surgery, i.e. because surgery had failed, was deemed inappropriate or contraindicated or was declined by the patient. Surgery was often deemed inappropriate because of the failure to localise the abnormal parathyroid gland, either reflecting the growing dependency of surgeons on preoperative localisation before surgery per se or, more likely, because the disease is being found earlier using biochemical screening. Earlier identification leads to a rise in the number of borderline cases being uncovered, which means that surgeons are using preoperative localisation to manage the disease appropriately i.e. treat with drugs as an alternative to surgery. In a few cases, patients were treated to resolve borderline hypercalcaemia, and in 43 patients (27 (63\%) of whom subsequently underwent surgery) the reason for treatment was to reduce calcium levels in preparation for parathyroidectomy; neither of these are labelled indications for the product. Although the recommended starting dose for PHPT patients in the product label is $30 \mathrm{mg}$ twice daily,

Table 3 Adverse drug reactions (ADRs) by MedDRA system organ class (SOC) and preferred term reported by $\geq 3$ patients (full analysis set, $n=303$ ).

MedDRA SOC and preferred term

Number of patients reporting $(\%)$

Gastrointestinal disorders

Nausea

Vomiting

Abdominal pain

Diarrhoea

Dyspepsia

Nervous system disorders

Paraesthaesia

Dizziness

Headache

Tremor

Metabolism and nutrition disorders

Hypocalcaemia

Decreased appetite

Ear and labyrinth disorders

Vertigo

Investigations

Blood magnesium decreased

$41(13.5)$

$11(3.6)$

$6(2.0)$

$6(2.0)$

$3(1.0)$

5 (1.7)

$3(1.0)$

$3(1.0)$

$3(1.0)$

5 (1.7)

$4(1.3)$

$5(1.7)$

$3(1.0)$

MedDRA, Medical Dictionary for Regulatory Activities. 
(a)

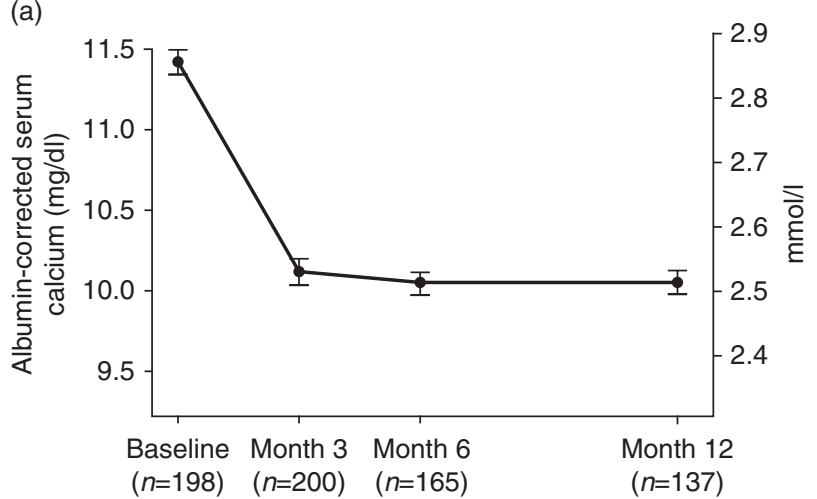

(b)
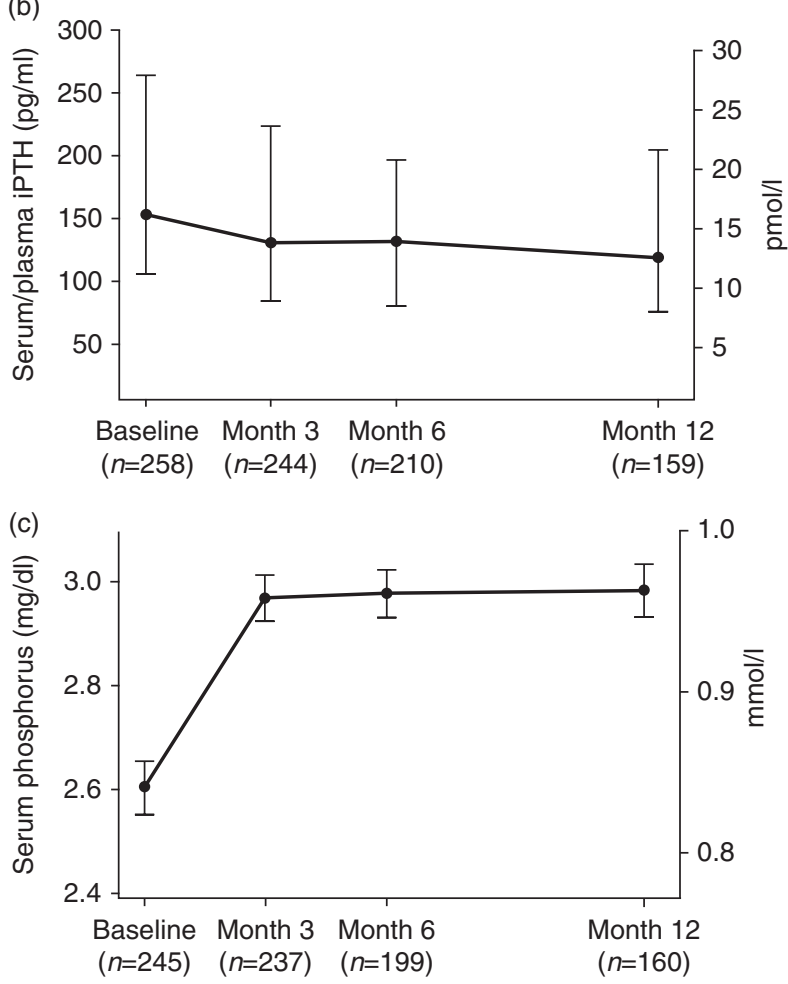

\section{Figure 2}

Concentrations of laboratory calcium, intact parathyroid hormone (iPTH) and phosphorus at each time point: (a) albumin-corrected serum calcium (mg/dl) (mean and s.E.M.); (b) plasma or serum iPTH (pg/ml) (median and IQR); and (c) serum phosphorus (mg/dl) (mean and s.E.M.).

only $44 \%$ of patients in our sample received this dose, with $54 \%$ of patients starting on a lower dose (most commonly $30 \mathrm{mg}$ once daily). Post hoc analysis showed that the mean baseline calcium was slightly lower in those on $30 \mathrm{mg}$ once daily than $30 \mathrm{mg}$ twice daily (albumin-corrected values of 2.78 and $2.91 \mathrm{mmol} / 1$ respectively), but it is not clear whether this measure influenced the choice of starting dosage. A once-daily dose of $30 \mathrm{mg}$ was also the most common starting dose in a recent observational study in Italian patients (7). In spite of this, we observed clinically relevant reductions in calcium and iPTH levels after cinacalcet treatment started, as well as partial correction of hypophosphataemia. These are important outcomes in this patient population and our findings are consistent with the results of randomised placebocontrolled trials $(8,9)$ as well as other studies $(7,10,11)$.

At baseline, patients typically had mild-to-moderate hypercalcaemia, with mean ACSC concentration of $11.4 \mathrm{mg} / \mathrm{dl}(2.9 \mathrm{mmol} / \mathrm{l})$. Three months after starting cinacalcet treatment, more than one-half of evaluable patients (56\%) achieved a reduction in ACSC concentration from the baseline of at least $1 \mathrm{mg} / \mathrm{dl}(0.25 \mathrm{mmol} / \mathrm{l})$, and similar proportions achieved this target at 6 and 12 months. This degree of change is likely to be of clinical relevance, given that in patients with asymptomatic PHPT, management guidelines recommend surgery when the concentration of serum calcium is $1 \mathrm{mg} / \mathrm{dl}$ or more above the upper limit of normal $(10.5 \mathrm{mg} / \mathrm{dl}$ (2.63 mmol/l)) (2). Nearly two-thirds of evaluable patients had an ACSC concentration of $\leq 10.3 \mathrm{mg} / \mathrm{dl}$ ( $\leq 2.6 \mathrm{mmol} / \mathrm{l})$ at 3 months, and the corresponding proportions at 6 and 12 months were similar. As shown in Fig. 1b, the proportions at these three time points were much higher than at baseline. The results for total calcium concentrations over time were similar to those for albumin-corrected values.

A notable finding in this study was that bone pain and kidney stones were much more commonly reported as presenting symptoms than expected, because most cases of mild-to-moderate PHPT are asymptomatic. This high prevalence might have been due to the highly selected group of patients enrolled in our study, in whom surgery had either failed or was inappropriate for some reason. We also found that in more than $40 \%$ of the patients for whom surgery was not considered appropriate by the physician, the reason given was that the diseased parathyroid gland could not be located. This is surprisingly high, given the reported sensitivity of localising methods such as high-resolution ultrasonography, technetium (Tc) 99m-methoxyisobutylisonitrile parathyroid scintigraphy and magnetic resonance imaging. These three techniques correctly identified 90, 72 and $67 \%$ of adenomas, respectively, in a study of PHPT patients, and combining the methods improved sensitivity even further (12). It is possible that these specialised techniques were not readily available; alternatively, as mentioned above, patient selection criteria might have been a factor, or the 
physician might have had additional reasons for choosing cinacalcet for these patients that were not reported in the eCRF.

Neither bone mineral density (BMD) data nor bone turnover marker data are presented in this paper. A few patients had BMD data at baseline and a later time point (obtained by dual energy X-ray absorptiometry scanning), but nearly all these data were at the spine and hip, with only one patient having data at the radius. Evidently, BMD measurement was not a common assessment in patients with PHPT in Europe, even though it is recommended in recent international guidelines (2). In this patient population, bone loss is primarily at the cortical site, so BMD measurements at the forearm would have been more informative.

Adverse reactions to cinacalcet treatment were mainly mild or moderate, with gastrointestinal disorders, primarily nausea being the most common events reported. Hypocalcaemia occurred in five patients: in one of these patients, the event was classed as serious, but resolved after drug withdrawal. There were no unexpected safety concerns. The tolerability profile in our sample was similar to that seen in a placebo-controlled study of cinacalcet (8).

The PRIMARA study shares the limitations of observational analyses, e.g. the lack of a control group and a limited ability to control for variables. To assess the effect of selection bias, we collected reasons for non-enrollment of 77 patients who were considered as study participants: most failed because they used cinacalcet for more than 1 month before enrollment. As the centres which participated in the study were chosen for their experience in treating subjects with PHPT and use of cinacalcet, it is possible that they might not be a representative of other clinics in the participating countries. A post hoc comparison of baseline characteristics and study outcomes by centre type did not show marked differences between university hospital and general hospitals, and there were too few private practice centres to allow meaningful comparison. An additional potential bias might have resulted from the inclusion in the main analysis set (FAS) of post-surgical data from 45 patients who underwent parathyroidectomy, 43 of whom discontinued cinacalcet before surgery; to address this, we also analysed results in the 'FAS with Censoring' set, which excludes data after cinacalcet discontinuation, and found that calcium outcomes were similar in the two analysis sets.

The patients included in our study appear to be the representative of the general population of patients with PHPT, for whom cinacalcet therapy might typically be considered. Although no predefined criteria were used to guide the investigators in diagnosing PHPT, the hormone and mineral profile of patients at baseline strongly suggest that PHPT was an accurate diagnosis in this study sample. However, as patients were enrolled at different sites across the European Union, physicians might have been influenced by different local guidelines for treatment of PHPT; for example, they might have used a different threshold for initiating PHPT treatment, whether medically with cinacalcet or with parathyroidectomy. In any case, it should be noted that the study was not designed to inform recommendations on how best to use cinacalcet in this patient population, and further studies are necessary to confirm the potential benefits of treatment shown by our trial.

\section{Supplementary data}

This is linked to the online version of the paper at http://dx.doi.org/10.1530/ EJE-14-0355.

\section{Declaration of interest}

L C Hofbauer has received honoraria for advisory boards and lectures from Amgen, Merck and Novartis; M Farouk and C Mattin are employees of Amgen (who funded the study), and have stock options in Amgen. M Muñoz Torres has received lecture fees from Amgen, Eli Lilly and Co. and Alexion. All other authors have nothing to disclose.

\section{Funding}

The study was funded by Amgen.

\section{Acknowledgements}

The authors thank Dr Filomena Cetani, Department of Clinical and Experimental Medicine, University of Pisa, Italy, for valuable help with patient follow-up. Medical writing support was provided by Roger Nutter (Bioscript Stirling Ltd) and funded by Amgen (Europe) $\mathrm{GmbH}$.

\section{References}

1 Marcocci C \& Cetani F. Primary hyperparathyroidism. New England Journal of Medicine 2011365 2389-2397. (doi:10.1056/ NEJMcp1106636)

2 Bilezikian JP, Brandi ML, Eastell R, Silverberg SJ, Udelsman R, Marcocci C \& Potts JT Jr. Guidelines for the management of asymptomatic primary hyperparathyroidism: summary statement from the Fourth International Workshop. Journal of Clinical Endocrinology and Metabolism 201499 3561-3569. (doi:10.1210/jc.2014-1413)

3 Nemeth EF \& Bennett SA. Tricking the parathyroid gland with novel calcimimetic agents. Nephrology, Dialysis, Transplantation 199813 1923-1925. (doi:10.1093/ndt/13.8.1923)

4 Nemeth EF, Heaton WH, Miller M, Fox J, Balandrin MF, Van Wagenen BC, Colloton M, Karbon W, Scherrer J, Shatzen E et al. Pharmacodynamics of the type II calcimimetic compound cinacalcet 
HCl. Journal of Pharmacology and Experimental Therapeutics 2004308 627-635. (doi:10.1124/jpet.103.057273)

5 Coburn JW, Elangovan L, Goodman WG \& Frazaõ JM. Calcium-sensing receptor and calcimimetic agents. Kidney International. Supplement 1999 73 S52-S58. (doi:10.1046/j.1523-1755.1999.07303.x)

6 Endres DB \& Rude RK. Mineral and bone metabolism. In Tietz Textbook of Clinical Chemistry, edn 3rd, pp 1395-1406. Eds CA Burtis \& ER Ashwood, Philadelphia: WB Saunders Company, 1999.

7 Saponaro F, Faggiano A, Grimaldi F, Borretta G, Brandi ML, Minisola S, Frasoldati A, Papini E, Scillitani A, Banti C et al. Cinacalcet in the management of primary hyperparathyroidism: post marketing experience of an Italian multicentre group. Clinical Endocrinology 2013 79 20-26. (doi:10.1111/cen.12108)

8 Peacock M, Bilezikian JP, Klassen PS, Guo MD, Turner SA \& Shoback D. Cinacalcet hydrochloride maintains long-term normocalcemia in patients with primary hyperparathyroidism. Journal of Clinical Endocrinology and Metabolism 200590 135-141. (doi:10.1210/jc. 2004-0842)
9 Shoback DM, Bilezikian JP, Turner SA, McCary LC, Guo MD \& Peacock M. The calcimimetic cinacalcet normalizes serum calcium in subjects with primary hyperparathyroidism. Journal of Clinical Endocrinology and Metabolism 200388 5644-5649. (doi:10.1210/jc.2002-021597)

10 Cetani F, Saponaro F, Banti C, Cianferotti L, Vignali E, Chiavistelli S, Viccica G, Pinchera A \& Marcocci C. Cinacalcet efficacy in patients with moderately severe primary hyperparathyroidism according to the European Medicine Agency prescription labeling. Journal of Endocrinological Investigation 201235 655-660. (doi:10.3275/7970)

11 Marcocci C, Chanson P, Shoback D, Bilezikian J, Fernandez-Cruz L, Orgiazzi J, Henzen C, Cheng S, Sterling LR, Lu J et al. Cinacalcet reduces serum calcium concentrations in patients with intractable primary hyperparathyroidism. Journal of Clinical Endocrinology and Metabolism 200994 2766-2772. (doi:10.1210/jc.2008-2640)

12 Cakal E, Cakir E, Dilli A, Colak N, Unsal I, Aslan MS, Karbek B, Ozbek M, Kilic M, Delibasi T et al. Parathyroid adenoma screening efficacies of different imaging tools and factors affecting the success rates. Clinical Imaging 201236 688-994. (doi:10.1016/j.clinimag.2012.01.038)

Received 1 May 2014

Revised version received 17 September 2014

Accepted 19 September 2014 